Revue des patrimoines

34 | 2018

Lieux de pouvoirs. Architectures administratives dans la France contemporaine, 1945-2013. Avant/Après la décentralisation

\title{
Les Archives départementales du Val-de-Marne : un bâtiment structurant de l'administration du nouveau département
}

The Departmental Archives of the Val-de-Marne, Building the Administration of a New Department

Marie-Andrée Corcuff

\section{(2) OpenEdition}

\section{Journals}

Édition électronique

URL : http://journals.openedition.org/insitu/16077

DOI : 10.4000/insitu. 16077

ISSN : 1630-7305

Éditeur

Ministère de la culture

Référence électronique

Marie-Andrée Corcuff, « Les Archives départementales du Val-de-Marne : un bâtiment structurant de l'administration du nouveau département », In Situ [En ligne], 34 | 2018, mis en ligne le 17 juillet 2018, consulté le 20 avril 2019. URL : http://journals.openedition.org/insitu/16077 ; DOI : 10.4000/ insitu. 16077

Ce document a été généré automatiquement le 20 avril 2019.

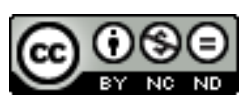

In Situ Revues des patrimoines est mis à disposition selon les termes de la licence Creative Commons Attribution - Pas d'Utilisation Commerciale - Pas de Modification 4.0 International. 
Les Archives départementales du Val-de-Marne : un bâtiment structurant de l'administration du nouveau département

The Departmental Archives of the Val-de-Marne, Building the Administration of a New Department

Marie-Andrée Corcuff

Introduction

(fig. 1) 


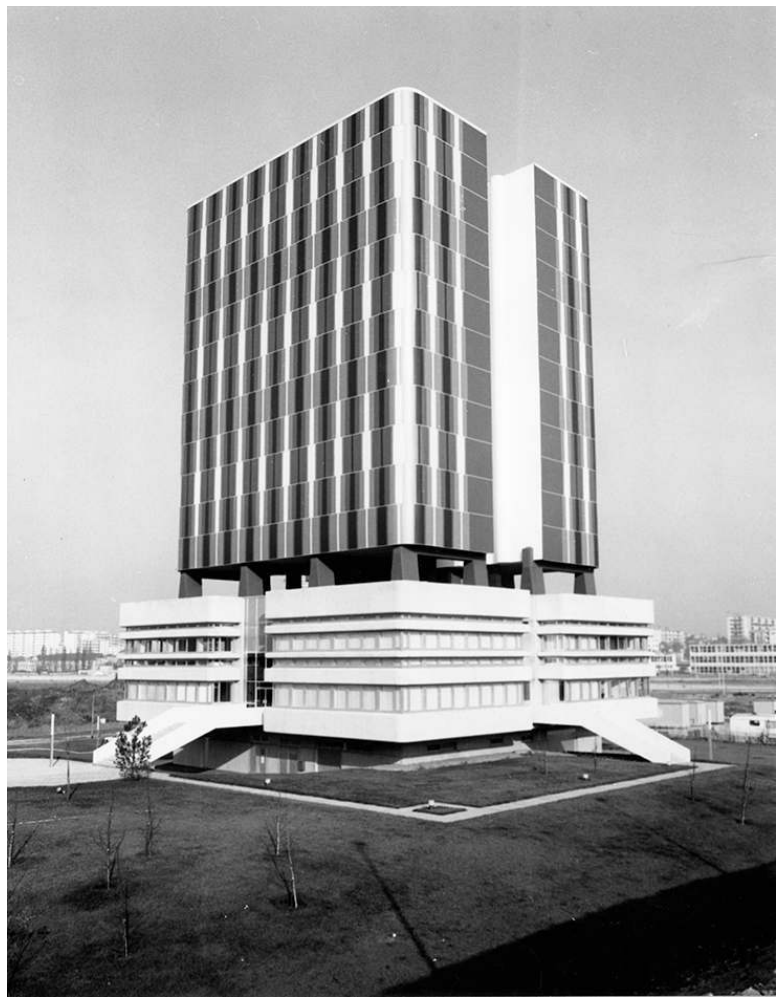

Le bâtiment achevé : une silhouette signature d'un quartier.

(c) Archives départementales du Val-de-Marne, 16FI 28_162.

2 «Le bâtiment d'archives constitue un programme qui n’a été jusqu'ici que fort peu exploré par les historiens de l'architecture ». Ainsi commence le compte rendu de lecture que fait Alice Thomine pour la Bibliothèque de l'École des chartes en 2007, au sujet du numéro 10 de la revue Livraisons d'histoire de l'architecture et des arts qui s'y rattachent ${ }^{1}$, consacré précisément aux bâtiments d'archives ${ }^{2}$. Les différentes contributions à ces Livraisons explorent le champ historique, proposent des clés de compréhension et esquissent des pistes de recherche pour poursuivre, notamment à travers les trois études monographiques présentées, qui concernent des situations très différentes: les archives départementales de la Meuse à Bar-le-Duc, les archives de l'ancien département de la Seine et de la Ville de Paris, le Centre des Archives du monde du travail à Roubaix.

3 Avant de s'intéresser au bâtiment des archives départementales du Val-de-Marne, qui fait l'objet de cette introduction à l'analyse architecturale qu'en propose Franca Malservisi', sans doute faut-il rappeler que le terme "archives" est ici particulièrement polysémique : bien sûr, il désigne, ici comme ailleurs, à la fois un contenu (les documents conservés), un contenant (le bâtiment), l'administration qui les gère (l'institution) mais aussi un quartier et une rue de Créteil. Le constater, c'est dire simplement la place que ce bâtiment occupe dans l'espace public cristolien et la force symbolique qui s'y attache depuis son édification, réalisée de 1972 à 1974. 


\section{Le bâtiment d'archives, une typologie architecturale qui s'impose en France dans la deuxième moitié du xx e siècle, sous l'égide de la direction des Archives de France (service technique)}

4 Si la loi du 5 brumaire an $\mathrm{V}(26$ octobre 1796$)$ instaure dans chaque chef-lieu de département un « dépôt » d'archives, il faut attendre le $\mathrm{xx}^{\mathrm{e}}$ siècle pour qu'une typologie architecturale dédiée à la conservation des archives commence à se dégager visiblement dans les villes chef-lieu de département. Les débuts sont timides : on ne dénombre de 1918 à 1939 qu'une douzaine de constructions de dépôts d'archives départementales, dont la conception assez sommaire reflète presque uniquement des préoccupations de stockage. L'étude monographique publiée par Stéphanie Quantin sur les archives de l'ancien département de la Seine et de la Ville de Paris s'est attachée à analyser les différentes facettes de cette architecture en recherche d'identité au tournant des XIX et $\mathrm{xx}^{\mathrm{e}}$ siècles $^{4}$, appliquée au cas très particulier des archives parisiennes.

5 Au début des années 1950, dans la mouvance des grands chantiers de reconstruction d'après-guerre et de l'aménagement du territoire, y compris dans le champ culturel, qui voit notamment les bibliothèques, les musées et les théâtres se multiplier, les archives font l'objet de gros efforts d'équipement. Cette dynamique est lancée sous l'impulsion de Charles Braibant (1889-1976), directeur général des Archives de France de 1948 à 1959 : construction de 16 bâtiments neufs, réaménagement ou extension de 25 bâtiments existants. Dès 1948, la direction des Archives de France s'est dotée de l'outil qui va lui permettre de construire sur ce sujet une véritable expertise, le service technique, dont la responsabilité est successivement confiée à Guy Duboscq ${ }^{5}$, à François Dousset (1911-1991), puis en 1956 à Michel Duchein ${ }^{6}$.

6 C'est ce dernier qui donne au service technique de la direction des Archives de France toute sa dimension et son rayonnement en matière d'élaboration et de mise en œuvre des normes archivistiques, et particulièrement celles qui s'appliquent à la construction des bâtiments d'archives. Le corpus, qui s'est considérablement étoffé depuis les années 1960, encadre toujours la thématique.

7 Michel Duchein s'appuie alors sur une équipe resserrée de conservateurs et s'entoure des conseils d'architectes et de bibliothécaires pour étudier les problèmes techniques spécifiquement attachés à la construction des bâtiments d'archives. En 1966 est publié le premier ouvrage de référence sur le sujet: Les Bâtiments d'archives ${ }^{7}$, fruit des premiers échanges et expériences conduits avec les maîtres d'ouvrage et les archivistes concernés, qui pose les fondements de ce qui va bientôt s'apparenter à une réglementation, même si Michel Duchein s'en défend. Force est de constater que ce corpus de normes en constitution prend progressivement valeur de référence et de cahier des charges pour tout projet de construction ou d'aménagement pour lequel les maîtres d'ouvrage sollicitent une subvention de l'État. En effet, depuis un arrêté du 20 juin 1950, «les constructions d'archives départementales et municipales en France peuvent bénéficier d'une subvention de l'État, moyennant le visa technique de la direction des Archives de France. " Jusqu'en 1970, dans l'organisation du tout jeune ministère de la Culture, l'instruction des attributions de subventions à la construction se fait sous l'égide du Conseil général des Bâtiments de France, placé auprès de la direction de l'Architecture, 
avec lequel le service technique de la direction des Archives de France entretient des relations étroites. Les échanges et débats méthodologiques et techniques qui résultent de cette proximité contribuent largement à façonner l'expertise du service technique sur ces sujets.

C'est ainsi qu'a pu être élaboré un "programme-type $»^{8}$, qui a servi de base à la construction ou au réaménagement d'une trentaine de bâtiments d'archives en France et à l'étranger dans les décennies 1960 et 1970. Y sont notamment définis les trois éléments constitutifs essentiels de ce qui est encore appelé à l'époque un « dépôt d'archives » : les magasins, les locaux de tri et de classement, les locaux de consultation (fig. 2).

Figure 2

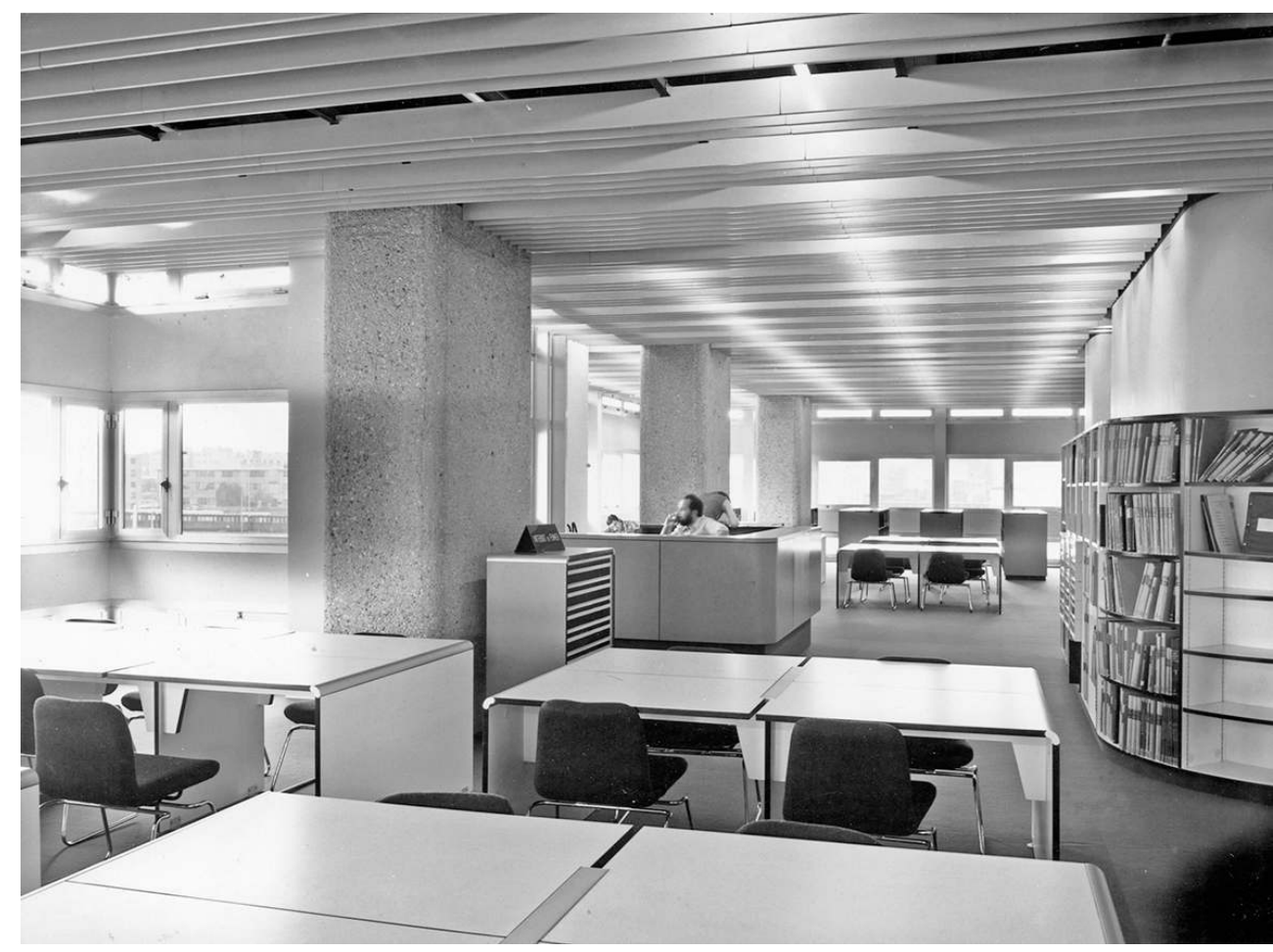

La salle de lecture.

(c) Archives départementales du Val-de-Marne, 16FI 28_583.

9 En effet, l'accueil des publics prend dès les années 1960 une dimension croissante dans les bâtiments d'archives, face à l'augmentation de la fréquentation et des sollicitations dont les archives sont de plus en plus l'objet (fig. 3). Le programme de construction des archives départementales du Val-de-Marne s'est appuyé sur ce modèle, comme on le verra plus loin ; néanmoins, maître d'ouvrage, maîtres d'œuvre et tutelle administrative ont su s'en affranchir suffisamment pour produire une réalisation encore saluée comme originale (fig. 4). 
Figure 3

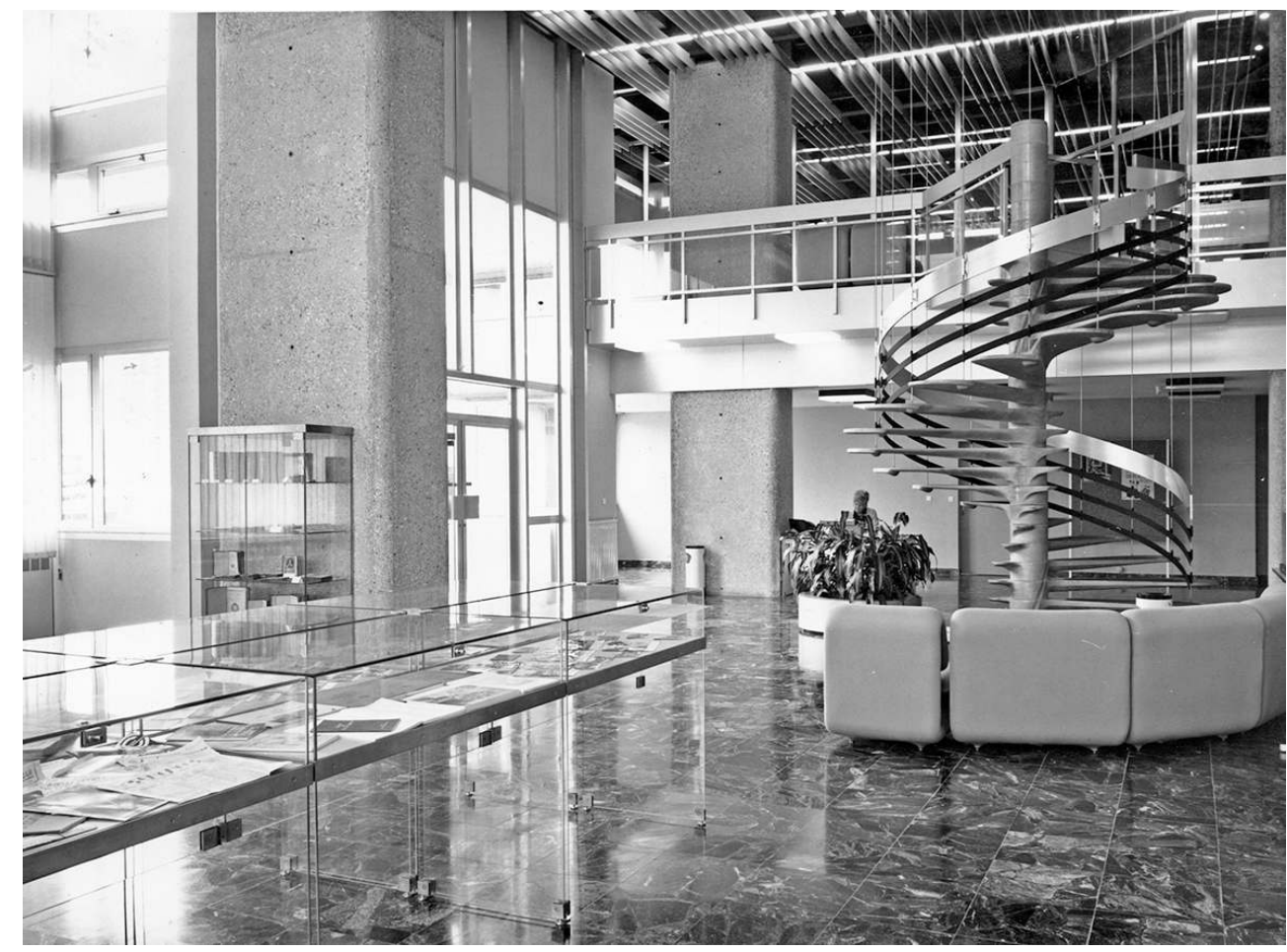

Le hall d'accueil et d'exposition

(c) Archives départementales du Val-de-Marne, 16FI 28_580. 


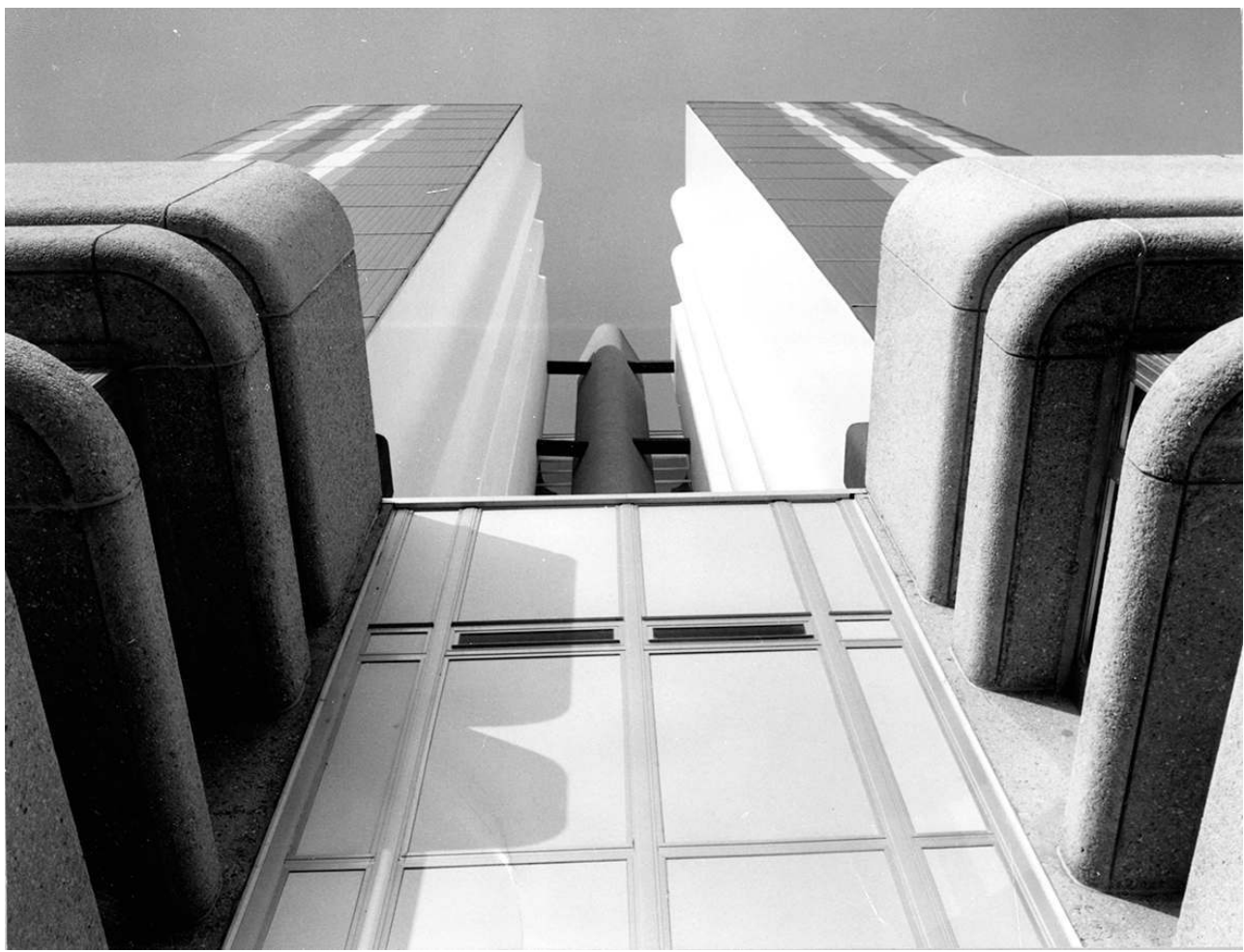

Contre-plongée à l'aplomb de la faille qui sépare les silos des magasins.

(c) Archives départementales du Val-de-Marne, 16FI 28_164.

\section{La loi du 10 juillet 1964 portant création des nouveaux départements de la région parisienne... et de leurs archives}

Sur le contexte qui a présidé à la reconfiguration géopolitique et administrative de la région parisienne, voulue par l'État dès les années 1950 dans le cadre de la planification, je renvoie à l'ouvrage collectif réalisé sous la direction scientifique d'Emmanuel Bellanger et de Julia Moro (en collaboration étroite avec les archives départementales) à l'occasion du $50^{\mathrm{e}}$ anniversaire de la loi du 10 juillet 1964. Il décline chacune des cinquante années d'existence du département du Val-de-Marne à travers le prisme des archives publiques et privées produites et collectées durant la période. On y lit notamment que «Le volontarisme de l'État a pour mot d'ordre : administrer, équiper et désenclaver la région capitale en densifiant les services publics ${ }^{9}$. Cet ouvrage s'inscrit aussi dans la continuité d'un séminaire de recherche, "Trajectoires historiques d'un département: le Val-deMarne, de sa création à nos jours. Gérer, administrer et aménager un territoire de banlieue ", mené de 2011 à 2013 par Julia Moro sous la direction de Florence Bourillon, professeure d'histoire contemporaine à l'université Paris-Est-Créteil et à l'époque directrice du Centre de recherche d'histoire européenne comparée ${ }^{10}$.

Les premiers services préfectoraux apparaissent peu à peu sur cette partie du territoire cristolien naguère dévolu au maraîchage: Paul Camous (1922-2017), premier préfet délégué de 1965 à 1967, a pour mission d'en organiser la préfiguration et la première 
installation, en étroite articulation avec la planification urbaine confiée à Pierre Dufau (1908-1985), l'architecte en chef du Nouveau Créteil. Franca Malservisi revient sur le contexte urbanistique local en s'appuyant sur l'ouvrage de référence que lui a consacré Laurent Coudroy de Lille en $2005^{11}$. Dans la liste des bâtiments publics inscrits dans la planification, puis la préfiguration, et enfin les calendriers de construction, celui des archives départementales se situe en bonne position.

Pour la direction des Archives de France aussi, la réorganisation de la région parisienne et la création de ces sept nouveaux départements représente à la fois un défi encore jamais rencontré et un terrain d'expérimentation propice au développement et à la structuration de son ingénierie de conseil auprès des maîtres d'ouvrage. Il s'agit en effet d'accompagner les nouveaux départements ${ }^{12}$ (Essonne, Hauts-de-Seine, Seine-Saint-Denis, Val-de-Marne, Val-d'Oise et dans une moindre mesure, Paris et les Yvelines) dans la conception et la construction ou l'aménagement d'un bâtiment pour les services d'archives nouvellement créés. Le Val-de-Marne est le premier à s'engager dans cette aventure avec l'appui résolu du premier préfet, Lucien Lanier (1919-2015), préfet du Valde-Marne de 1967 à 1974 (fig. 5).

Figure 5

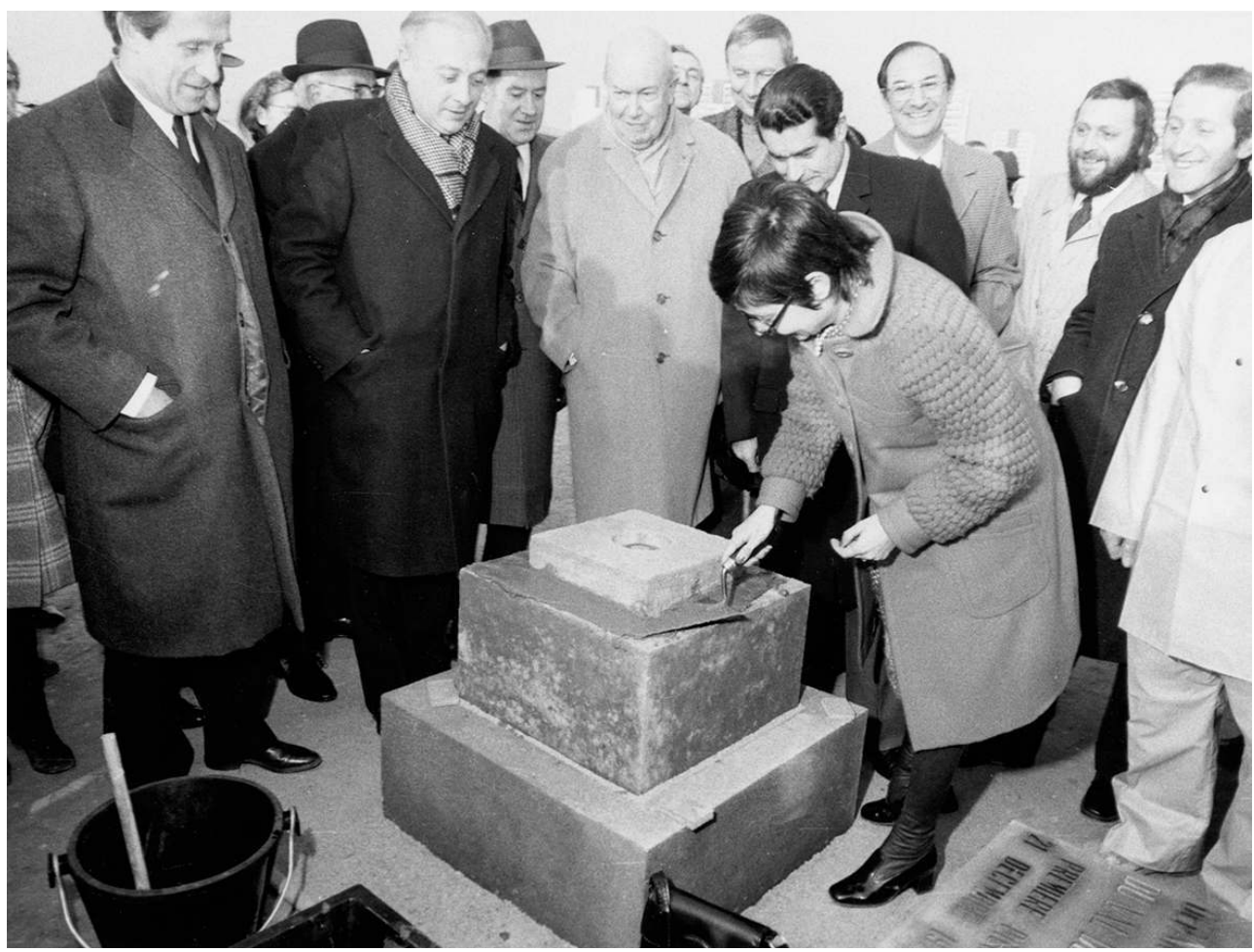

Pose de la première pierre du bâtiment des archives, 21 décembre 1972. Au premier plan : Claire Berche, au second plan : Daniel Badani, Roland Nungesser, Guy Duboscq, Lucien Lanier, Michel Duchein.

(c) Archives départementales du Val-de-Marne, 16FI 28_354. 


\section{Les Archives départementales du Val-de-Marne: partie intégrante de la nouvelle administration}

13 Nommé préfet délégué à la suite de Paul Camous, Lucien Lanier devient préfet de plein exercice en décembre 1967. Les archives font dès lors partie des chantiers publics dont il suit personnellement le dossier d'avancement: pour l'anecdote, il se plaisait encore à rappeler lors de la cérémonie des quarante ans de la préfecture, en décembre 2012, l'insistance avec laquelle il avait réclamé « un très bon archiviste » à la direction des Archives de France. Il fut entendu: le $1^{\mathrm{er}}$ mai 1968, la première directrice des archives départementales, Claire Guérin, prend ses fonctions avec pour feuille de route la mission totalement inédite de créer les archives du Val-de-Marne ${ }^{13}$.

Dès le 18 mai, dans un bref aparté lors d'une séance du conseil général, le préfet Lanier lui exprime son intention « de lui faire construire quelque chose ». Trois mois à peine après sa nomination, le 25 juillet 1968, Claire Guérin adresse au service technique de la direction des Archives de France un document intitulé « Programme pour la construction du dépôt d'archives du Val-de-Marne ", répondant point par point au programme-type élaboré par Michel Duchein et son équipe. Le rapport d'activité qu'elle rend l'année suivante au titre de l'année 1968 détaille la manière dont s'est déroulée la création du service des archives, constitué en sus d'elle-même d'un commis intérimaire, provisoirement installé aux Archives de Paris, rejoint en octobre par une documentaliste ${ }^{14}$. Après une recherche infructueuse de locaux à Créteil, les archives finissent par occuper, de 1969 à 1974, une maison de $470 \mathrm{~m}^{2}$ appartenant au conseil général, située 7 villa Vernier à Saint-Maur-des-Fossés, de l'autre côté du pont de Créteil (fig. 6), dans l'attente de la construction du futur bâtiment (fig. 7), auquel l'article de Franca Malservisi est consacré. 
Figure 6

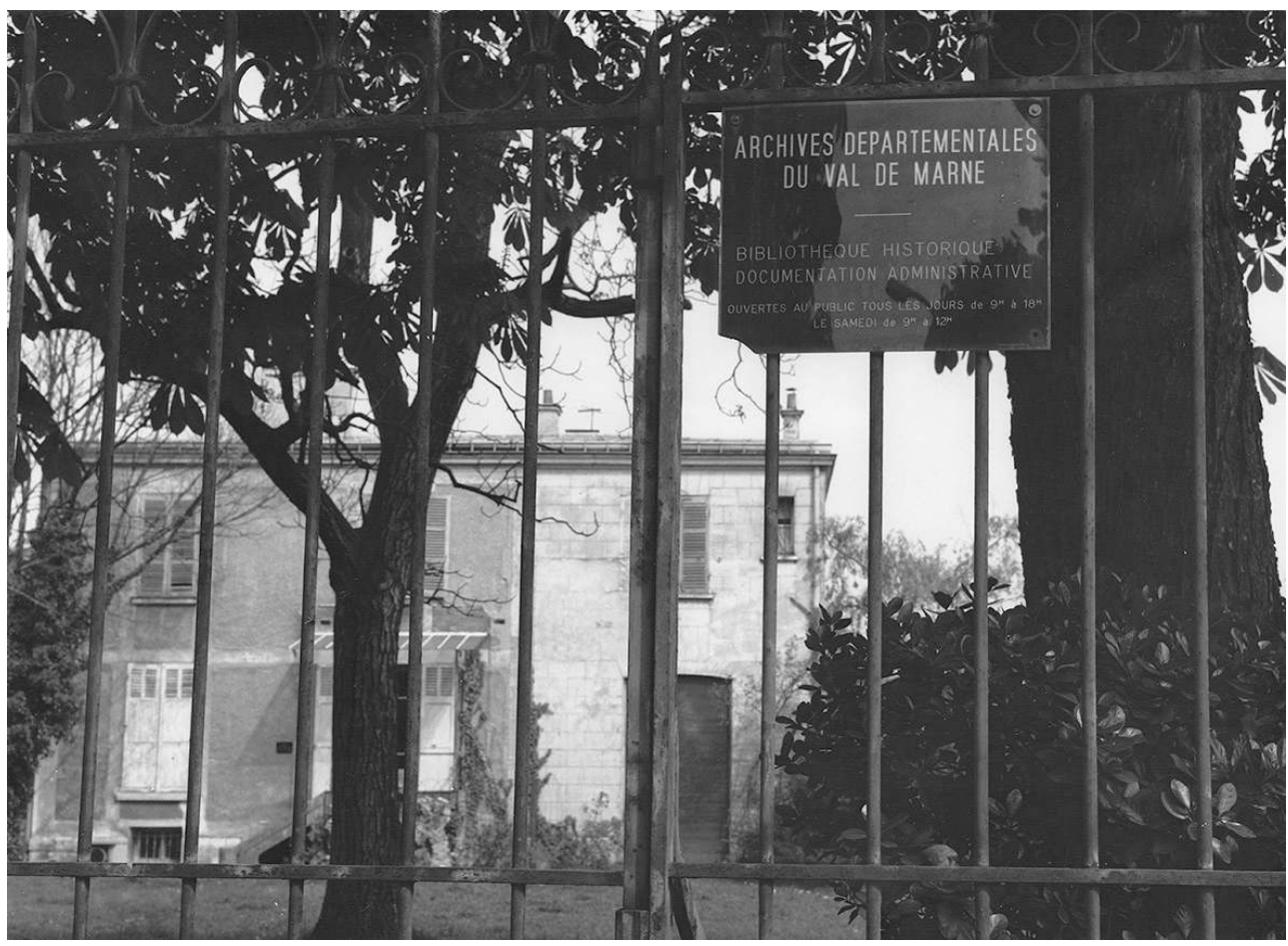

Les Archives départementales du Val-de-Marne à Saint-Maur-des-Fossés.

(c) Archives départementales du Val-de-Marne, AD94 2FI 68_617 (mai 1974) 


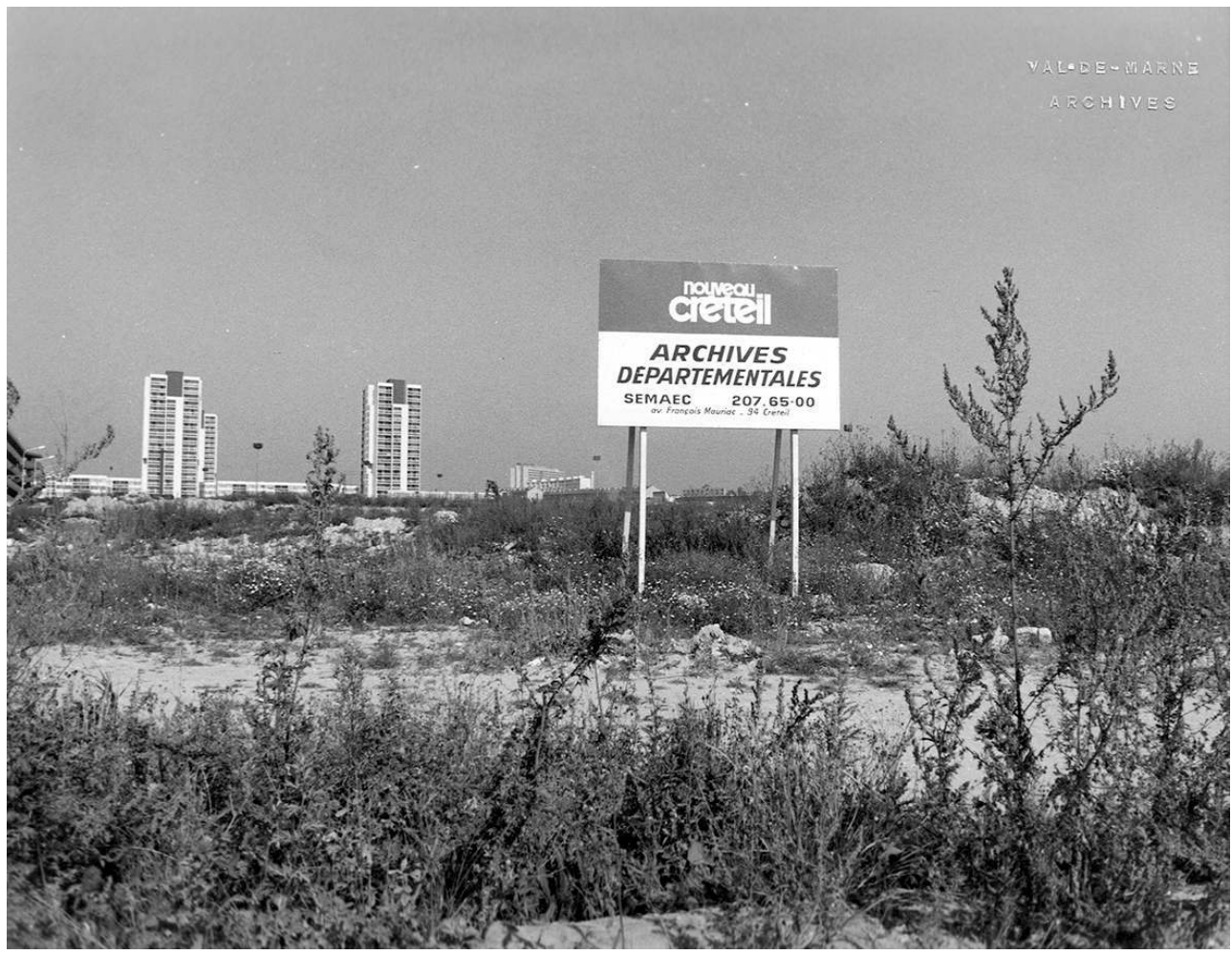

« Préfiguration » du futur bâtiment.

(c) Archives départementales du Val-de-Marne. 16FI 28_109.

15 Quarante-cinq ans après sa construction, la silhouette du bâtiment des archives départementales n'est plus un amer topographique dans le Nouveau Créteil: la forte densification urbaine lui a progressivement fait perdre ce rôle et gommé les points de vue panoramiques. En revanche, l'étrangeté de ces deux silos aveugles postés à l'entrée de la rue des Archives continue à intriguer le piéton égaré, qui finit toujours par venir y chercher des repères. Le bâtiment construit par l'agence Badani et Roux-Dorlut s'est finalement révélé à l'usage capable d'évoluer pour accompagner les mutations des archives: un récent chantier de restructuration des espaces dévolus au public et aux locaux professionnels et techniques (2010-2012) a permis de prendre en compte un certain nombre d'évolutions, principalement liées à la mise en accessibilité du site, à la diversification des publics, à la croissance de l'équipe et aux mutations des métiers. La programmation relative aux espaces de conservation, dans laquelle s'inscrit l'étude commandée en 2017 par le conseil départemental au conseil d'architecture, d'urbanisme et d'environnement (CAUE) du Val-de-Marne, va ouvrir une nouvelle page dans l'histoire du bâtiment. 


\section{NOTES}

1. - THOMINE, Alice. « Les bâtiments d'archives. Livraisons d'histoire de l'architecture et des arts qui s'y rattachent, $n^{\circ} 10$, rassemblées avec la collaboration d'Anne Georgeon-Liskenne et de Christian Hottin ». Bibliothèque de l'École des chartes, 2007, 165-1, p. 284-287. Voir le site : https:// www.persee.fr/doc/bec_0373-6237_2007_num_165_1_463498_t11_0284_0000_3 [consulté le 14/06/2018].

2. - LENIAUD, Jean-Michel (dir.). Les Bâtiments d'archives. Livraisons d'histoire de l'architecture et des arts qui s'y rattachent, $\mathrm{n}^{\circ}$ 10, 2005.

3. - MALSERVISI, Franca. "Le bâtiment des archives départementales du Val-de-Marne: un équipement du Nouveau Créteil de l'agence Badani et Roux-Dorlut », In Situ [En ligne], 34 | 2018, mis en ligne le 31 mai 2018, consulté le 14 juin 2018. URL: http://journals.openedition.org/ insitu/15699; DOI : 10.4000/insitu.15699.

4. - QUANTIN, Stéphanie. «Les Archives de l'ancien département de la Seine et de la Ville de Paris. À la recherche d'une architecture ». Livraisons d'histoire de l'architecture, $\mathrm{n}^{\circ} 10,2005$, p. 87-104; voir le site: https://www.persee.fr/doc/lha_1627-4970_2005_num_10_1_1017 [consulté le 14/06/2018].

5. - Guy Duboscq (1909-1994) a été directeur général des Archives de France de 1971 à 1975. Il participe à ce titre à la pose de la première pierre des archives du Val-de-Marne, le 21 décembre 1972.

6. - DUCHEIN, Michel. "Vingt ans de constructions... et l'avenir ». Dans Études d'archivistique 1957-1992. Recueil d'articles de Michel Duchein offert à l'auteur. Paris: Association des archivistes français, 1992, p.151-156. Voir le site: http://www.persee.fr/doc/ gazar_0016-5522_1992_hos_1_1_3262 [consulté le 14/06/2018].

7. - DUCHEIN, Michel. Les Bâtiments et équipements d'archives. Théorie et pratique des archives publiques en France. Paris : Conseil international des archives, 1966.

8. - Association des archivistes français. Manuel d'archivistique. Paris : Archives nationales, 1970, p. 569-604.

9. - BELLANGER, Emmanuel, MORO, Julia (dir.). Le Val-de-Marne, anthologie 1964-2014. Ivry-surSeine : Les Éditions de l'Atelier, 2014, p. 9-17.

10. - À consulter, regarder et écouter sur le site internet des archives du Val-de-Marne : http:// archives.valdemarne.fr/content/le-s\%C3\%A9minaire-trajectoires-historiques-dun-d\%C3\% A9partement [consulté le 14/06/2018].

11. - COUDROY de LILLE, Laurent. Une nouvelle ville : une histoire de l'urbanisme du Nouveau Créteil. Créteil : Institut d'urbanisme de Paris, 2005.

12. - AD Val-de-Marne, 1525W 27.

13. - Dans un témoignage recueilli par les archives départementales à l'occasion du $30^{\mathrm{e}}$ anniversaire de Mai 1968, Claire Berche (-Guérin) revient avec humour sur sa prise de fonction et ses premiers contacts avec le département (préfecture et conseil général) (AD Val-de-Marne, 1AV 161).

14. - AD Val-de-Marne, 1525W 1. 


\section{RÉSUMÉS}

Le bâtiment des archives départementales du Val-de-Marne a été construit de 1972 à 1974 par les architectes Daniel Badani et Michel Roux-Dorlut, sur un programme spécialement conçu pour cette utilisation fonctionnelle et doté d'une puissante visibilité dans l'urbanisme du Nouveau Créteil. Cette typologie architecturale attachée à la fonction "archives" s'est répandue en France dans la seconde moitié du xx $\mathrm{x}^{\mathrm{e}}$ siècle, sous l'égide de la direction des Archives de France. Fondé sur le principe de la stricte séparation des fonctions (locaux de conservation, locaux professionnels et techniques, locaux ouverts au public), le bâtiment des archives à Créteil est représentatif de la génération de bâtiments d'archives de l'époque de sa construction. La loi du 10 juillet 1964, en créant les nouveaux départements de la région parisienne, signe la naissance d'une nouvelle administration plus proche de la population, dont les archives départementales constituent un élément symbolique. Le Val-de-Marne est le premier des nouveaux départements à se doter d'un bâtiment d'archives, sous l'impulsion déterminante du premier préfet, Lucien Lanier, et de la première directrice, Claire Berche.

The archives building of the Val-de-Marne department was built between 1972 and 1974 to the designs of the architects Daniel Badani and Michel Roux-Dorlut. The building was specially designed for archival use and was also to be a strong symbol in the Nouveau Creteil town plan. Inspired by the central French Archives administration, departmental archive buildings were considered as an architectural model and many were constructed throughout France during the second part of the twentieth century. From the middle of the century, the building design stipulated separate premises for the different functions concerned, storage, offices for staff and public premises. Amongst the new archive buildings erected in the new departments circling Paris at that time, the Val-de-Marne building was the earliest. Lucien Lanier, the prefect of the department and Claire Berche, the first director of the archives, played a major role in the building's realisation.

\section{INDEX}

Mots-clés : archives départementales, Val-de-Marne, architecture du xxe siècle, Daniel Badani, Michel Roux-Dorlut, Claire Berche (Guérin), conservation, administration, Nouveau Créteil

Keywords : Departmental archives, Val-de-Marne, twentieth-century architecture, Danial Badani, Michel Roux-Dorlut, Claire Berche (Guérin), conservation, administration, Nouveau Créteil

\section{AUTEUR}

\section{MARIE-ANDRÉE CORCUFF}

Directrice des archives départementales du Val-de-Marne marie-andree.corcuff@valdemarne.fr 